\title{
MDCT in Diagnosis of Uncommon Fistulous Complications of Severe Pancreatitis-Pictorial Essay
}

\author{
Rajoo Ramachandran*, Prabhu Radhan, Subramanian Ilanchezhian, \\ Shubha Veppur Mohan, Mitesh Kumar \\ Department of Radiology, Sri Ramachandra University, Chennai, India \\ Email: drrajoor@gmail.com
}

Received 12 April 2014; revised 10 May 2014; accepted 17 May 2014

Copyright (C) 2014 by authors and Scientific Research Publishing Inc. This work is licensed under the Creative Commons Attribution International License (CC BY). http://creativecommons.org/licenses/by/4.0/

\begin{abstract}
Fistulous complication of pancreatitis is a surgical emergency with varied clinical presentations. Early diagnosis is very important to minimize morbidity whereas delayed or missed diagnosis can cause life threatening consequences. Multi detector computed tomography (MDCT) with and without intravenous contrast is an accurate and reliable imaging modality allowing prompt diagnosis and management. In this essay, we present an overview of the radiological features found in uncommon fistulous complications of pancreatitis.
\end{abstract}

\section{Keywords}

Pancreatitis, Pseudo Cyst, Pancreatic Fistula

\section{Introduction}

Fistula formation in pancreatitis is a rare complication observed in clinical practice and requires surgical intervention. By definition pancreatic fistula is defined as abnormal communication between the pancreas and the adjacent or distant organs, space and structures. The cause of pancreatic fistulas is the leakage of pancreatic secretions from disruption of the pancreatic duct due to pancreatic disease, surgery and trauma. To our knowledge, the incidence of spontaneous fistula formation in severe pancreatitis has not been reported so far in English literature.

Non enhanced and contrast enhanced computed tomography imaging with thin multi slice reconstruction is a noninvasive method for evaluation of such uncommon complications of pancreatitis. We present three cases of

*Corresponding author.

How to cite this paper: Ramachandran, R., Radhan, P., Ilanchezhian, S., Mohan, S.V. and Kumar, M. (2014) MDCT in Diagnosis of Uncommon Fistulous Complications of Severe Pancreatitis-Pictorial Essay. Surgical Science, 5, 237-241. 
fistulous complications of severe pancreatitis which was diagnosed preoperatively. The uncommon fistula formation which was diagnosed by CT includes, pancreatico-gastric fistula and pancreatico-colonic fistulas, (one involving the mid descending colon and one involving the splenic flexure)

\section{Cases with Detailed Description}

\subsection{Case 1}

A forty four years old male presented with upper abdominal pain associated with vomiting for the past one month. The vomitus contained food particles, was non bilious and was not blood stained. The pain was progressive in nature radiating to the back region. The laboratory investigations showed elevated total WBC (white blood cells) count and elevated serum amylase and lipase. Plain radiograph of the chest did not reveal underlying lung/pleural pathology. Non enhanced Computed Tomography (CT) of the abdomen showed complete replacement of pancreatic parenchyma by walled off necrosis. This necrotic collection had mottled appearance due to air pockets and demonstrated fistulous communication with the greater curvature of the stomach. Extensive surrounding inflammatory changes noted in the anterior para renal fascia on the left side (Figure 1(a), Figure 1(b) and Figure 1(c)). Intravenous contrast was not given because of altered renal function; hence the patient was subjected to Magnetic Resonance Imaging (MRI). T2 weighted Fat Saturation axial images confirmed the findings seen of CT scan demonstrating fistulous communication between the greater curvature of the stomach and pancreas (Figure 2(a), Figure 2(b) and Figure 2(c)).

\subsection{Case 2}

A forty two years old male presented with left sided abdominal pain for the past two and a half months. The pain was episodic in nature and associated with vomiting. He was diagnosed to have pancreatitis with retro peritoneal

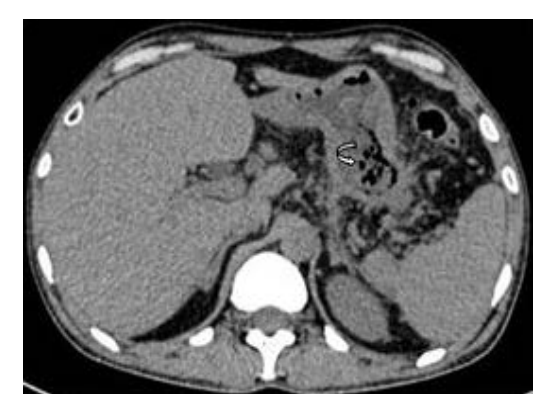

(a)

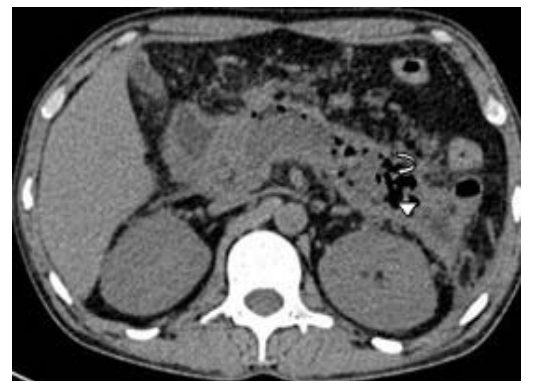

(b)

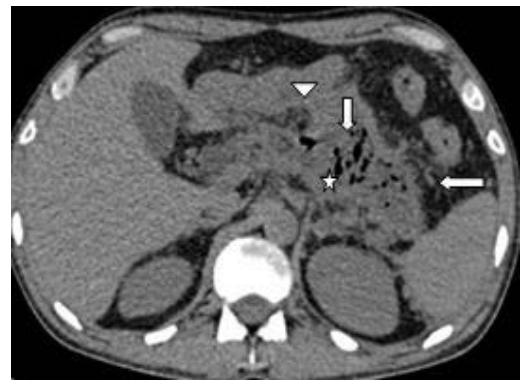

(c)

Figure 1. (a) (b) Non-enhanced axial CT of the abdomen showing mottled appearance due to air pockets in the fundus of the stomach (right curved arrow) and pancreatic parenchyma (left curved arrow). The mottled appearance within the stomach and the pancreas are suggestive of walled of necrosis. Thickening of the left anterior renal fascia is seen (arrow head); (c) Non-enhanced axial CT of the abdomen showing mottled appearance in the stomach(arrow head) and the pancreatic parenchyma (asterix) suggestive of fistulous communication between stomach and the pancreas(down arrow). Significant perigastric and peri pancreatic fat stranding is seen (left arrow).

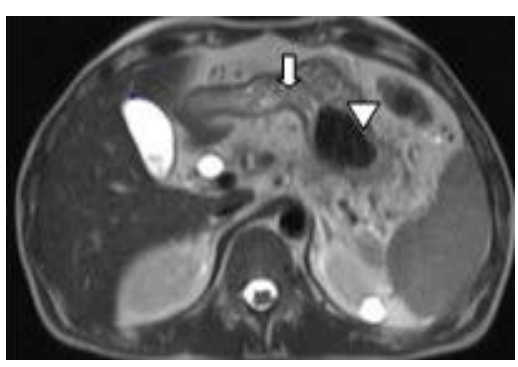

(a)

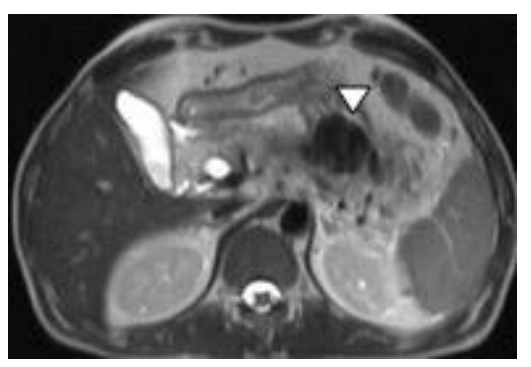

(b)

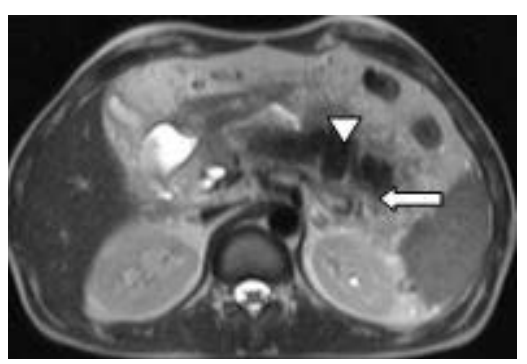

(c)

Figure 2. MRI T2 FAT SAT axial images shows fistulous communication between the stomach (down arrow) and pancreas (left arrow) as evidenced by large globules of air (seen as hypo intense areas) across the structures (arrow head). 
abscess four weeks ago for which he underwent laparoscopic abscess drainage. Presently, the patient complained of left sided abdominal pain and foul smelling loose stools 5 - 6 times/day. Blood investigations showed elevated total WBC count, elevated serum amylase and lipase levels. Contrast enhanced CT of the abdomen with contrast examination showed walled off necrosis of size $4.1 \times 7.5 \times 12.7 \mathrm{~cm}$ in the pancreatic region completely replacing the pancreatic parenchyma. The collection was seen to track along the anterior renal fascia and the lateral conal fascia. The collection had a fistulous communication with descending colon (Figure 3). Mesenteric fat stranding was noted surrounding the collection. Exploratory laparotomy showed a necrotic collection in the tail of pancreas which had a fistulous communication with the posterior wall of descending colon.

\subsection{Case 3}

A sixty three year old male presented with epigastric pain radiating to the back and associated with vomiting for the past four months. He had a few hospitalizations in the past due to recurrent alcoholic pancreatitis. Laboratory investigations showed elevated total WBC count, elevated serum amylase and lipase level. Serial blood investigations revealed drop in hemoglobin and packed cell volume. CT scan of whole abdomen with intravenous contrast revealed a large pseudocyst of size $4.1 \times 3.8 \times 4.6 \mathrm{~cm}$ in the pancreatic tail region extending upto and communicating with the splenic flexure of colon (Figure 4(a), Figure 4(b) and Figure 4(c)). Few hyper dense

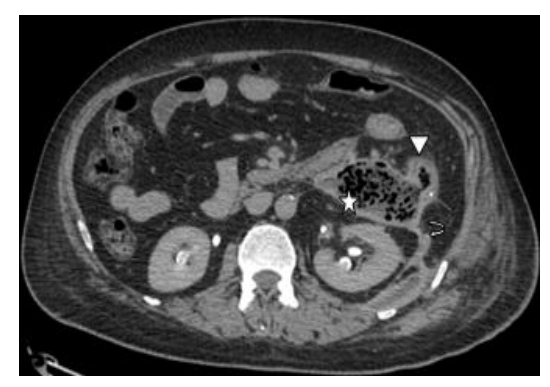

Figure 3. Contrast enhanced axial images of the abdomen in venous phase shows walled of necrosis with a fistulous communication (arrow) between the pancreas (asterix) and descending colon (arrow head). Significant colonic wall thickening noted. The left anterior renal fascia is thickened (curved arrow).
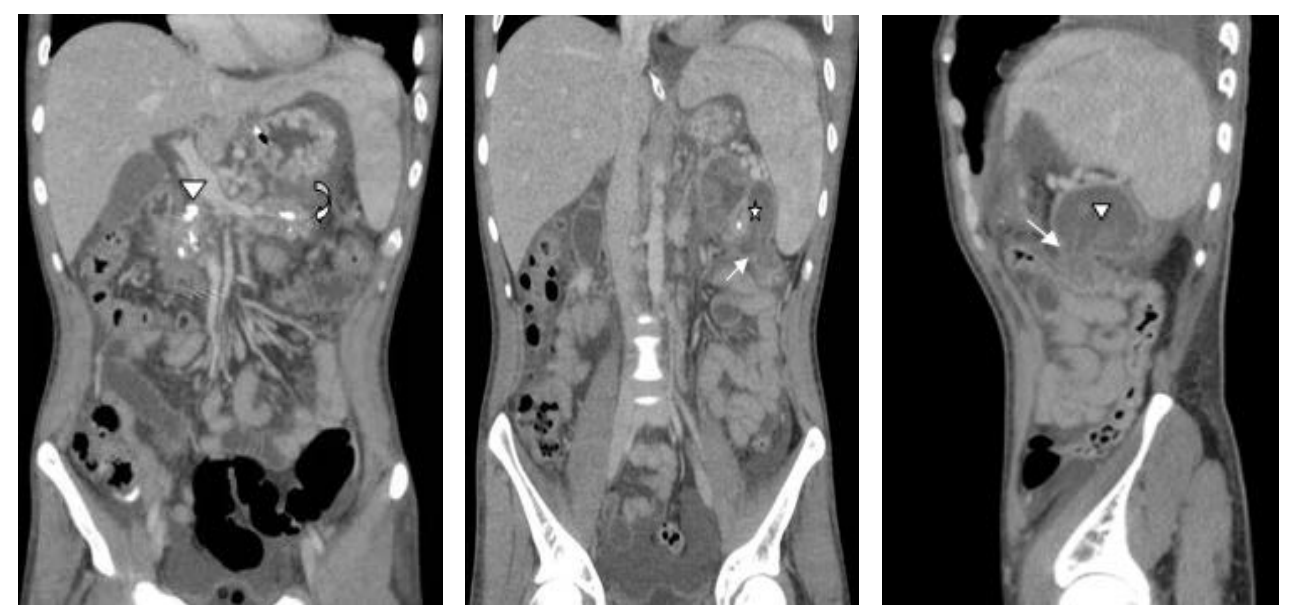

Figure 4. (a) Contrast enhanced CT abdomen in coronal view shows calcifications (arrow head) in the head and tail of the pancreas. Peripancreatic fat stranding and peri pancreatic fluid collection (curved arrow) is noted; (b) (c) Contrast enhanced CT abdomen in venous phase with reformated coronal and saggital view shows a large pseudocyst (asterix) which is seen to communicate with the splenic flexure (arrow). Few haemorrhagic components are noted within the pseudocyst (arrow head). 
areas were noted within the cyst suggestive of hemorrhagic component.

\section{Discussion}

Pancreatic fistulas (PF) are usually formed as a result of disruption of the pancreatic duct. Disruption of the pancreatic duct causes extravasations of pancreatic enzymes which forms a fluid collection. Persistence of fluid collection for a longer period (6 weeks), leads to the formation of a fibro inflammatory wall around the collection, which is otherwise known as a pseudocyst [1]. The common causes of pancreatic pseudocysts include acute pancreatitis, chronic pancreatitis, pancreatic neoplasm, post operative (pancreatoduodenectomy, distal pancreatectomy) and trauma. The incidence of pseudocyst is $12 \%-55 \%$ in acute pancreatitis and $20 \%-40 \%$ in case of chronic pancreatitis [2].

Several authors have suggested the possible pathophysiology for the development of fistulas in severe pancreatitis. It is suggested that erosion into the adjacent hollow viscous is mainly caused by digestion of the visceral wall by the pancreatic enzymes and pressure necrosis caused by the expanding cyst [3] [4]. A study done by Mehta et al. showed a cyst of size greater than $7.5 \mathrm{~cm}$ and volume $>250 \mathrm{ml}$ is more prone for rupture and requires surgical interventions [5].

The clinical features of internal PF depend upon the size of the fistulas and the location of the affected organs. The common symptoms caused by fistulization into the bowel include fever and features of sepsis suggesting infected fluid collection. The other features include abdominal pain, abdominal swelling, anorexia, weight loss and severe malnutrition.

The presence of amylase rich fluid in ascites is a hall mark of PF. A fluid amylase level which is five times greater than serum amylase level is highly indicative of PF [1].

Fistulous communication between the pancreas and the duodenum, jejunum and colon has been reported in previous studies done by Jai et al. [6]. Fistulous communication between the pancreas and the stomach were found to be very rare. Only a few cases of uncomplicated rupture of pseudocyst have been reported [7], like the ones reported here.

The main imaging modalities which can be used to identify PF include, CT, MRCP and ERCP. USG is useful to demonstrate the presence of ascites and plain radiography to identify pleural effusion.

The limitation in ERCP is that any fistula beyond the second part of duodenum could not be appreciated. The limitation of MRCP is mainly attributed to low spatial resolution, high time consuming and cost when compared to MDCT. Further MRCP will only demonstrate endogenous ductal fluid collection. The pancreatico-colonic fistula can be detected by CT with rectal water soluble contrast medium described by Tuney et al. [8].

Computed Tomography is highly accurate and an effective imaging modality for diagnosing PF. Abdominal CT imaging with or without oral \& intravenous contrast can be done, depending upon radiologist preference. CT imaging has the advantage of direct visualization of the pancreas, peripancreatic region and other intra-abdominal structures.

The features of pancreatic fistulas on cross sectional imaging include, walled of collection with air bubbles in the region of pancreas, communication of this collection with adjacent bowel loop, thickening of the involved bowel or colon involved. The other ancillary findings observed include, peri pancreatic fat stranding, ascites, peritonitis, bowel obstruction and thickening of the renal fascia.

Other complication of severe pancreatitis include communication with peritoneal, pleural cavities, bronchial tree [9] or development of fluid collections in the lesser sac, retroperitoneum, posterior mediastinum and thrombosis or bleeding of adjacent vascular structures.

\section{Conclusion}

There is often a delay in the diagnosis of such rare complications. The Radiologist must be aware of various clinical and radiological features of such uncommon complications which aid in faster diagnosis and optimize treatment. The advantage of CT is that it can noninvasively demonstrate pancreatic pathology and the fistulas in a single scan are useful when Endoscopic Retrograde Cholangiopancreatography (ERCP) does not provide adequate information [10].

\section{Acknowledgments}

The author would like to thank his father, mother, wife and friends for their moral support and patience. 


\section{Competing Interest}

The authors declare that there is no competing interest.

\section{Consent}

Oral consent obtained from relatives of the patients.

\section{References}

[1] Vege, S.S., Kendrick, M.L., Whitcomb, D.C., Ashley, S.W. and Grover, S. (2012) Clinical Features, Diagnosis, and Prevention of Pancreatic Fistulas. 25 July 2012, Up to Date.

[2] Apostolou, C., Krige, J.E.J. and Bornman, P.C. (2006) Pancreatic Pseudocysts. South African Journal of Surgery, 4, 148-155.

[3] Torres, W.E., Clements Jr., J.L., Sones, P.J. and Konpf, D.R. (1981) Gas in the Pancreatic Bed without Abscess. American Journal of Roentgenology, 137, 1131-1133.

[4] Clements Jr., J.L., Bradley III, E.L. and Eaton Jr., S.B. (1976) Spontaneous Internal Drainage of Pancreatic Pseudocysts. American Journal of Roentgenology, 126, 985-991. http://dx.doi.org/10.2214/ajr.126.5.985

[5] Mehta, R. and Suva Rana, D. (2004) Natural Course of Asymptomatic Pancreatic Pseudocyst: A Prospective Study. Indian Journal of Gastroenterology, 23, 140-142.

[6] Wig, J.D., Kochhar, R., Bharathy, K.G., Kudari, A.K., Doley, R.P., Yadav, T.D. and Kalra, N. (2008) Emphysematous Pancreatitis. Radiological Curiosity or a Cause for Concern? Journal of Pancreas, 9, 160-166.

[7] Willard, M.R. and Schafer, H.A. (1982) Resolution of Pancreatic Pseudo-Cyst by Spontaneous Rupture into Stomach. Southern Medical Journal, 75, 618-620. http://dx.doi.org/10.1097/00007611-198205000-00033

[8] Tüney, D., Altun, E., Barlas, A. and Yegen, C. (2008) Pancreatico-Colonic Fistula after Acute Necrotizing Pancreatitis. Diagnosis with Spiral CT Using Rectal Water Soluble Contrast Media. Journal of the Pancreas, 9, 26-29.

[9] Fekete, F., Le Mee, J., Langonnet, F., Gayet, B. and Belghiti, J. (1989) A Surgical Etiology of Respiratory Distress in Necrotizing Pancreatitis: Pancreato-Bronchial Fistula. 3 Cases. Chirurgie, 115, 106-112.

[10] Jain, S.K., Basra, B.K., Nanda, G., Srivathsan, R. and Gaza, R.C. (2009) Rare Case of Internal Pancreatic Fistula in a Young Adult Presenting with Massive Bilateral Pleural Effusion. BMJ Case Reports.

\section{Abbreviations}

MDCT: Multi detector Computed Tomography

MRCP: Magnetic Resonance Cholangiopancreatography

ERCP: Endoscopic Retrograde Cholangiopancreatography

PF: Pancreatic Fistulas 